\title{
BMJ Open Effectiveness of custom-made foot orthoses in patients with systemic lupus erythaematosus: protocol for a randomised controlled trial
}

\author{
María Reina-Bueno (D) , María del Carmen Vázquez-Bautista, \\ Inmaculada C Palomo-Toucedo, Gabriel Domínguez-Maldonado, \\ José Manuel Castillo-López, Javier Ramos-Ortega, Pedro V Munuera-Martínez
}

To cite: Reina-Bueno M, Vázquez-Bautista MdC, Palomo-Toucedo IC, et al. Effectiveness of custom-made foot orthoses in patients with systemic lupus erythaematosus: protocol for a randomised controlled trial. BMJ Open 2021;11:e042627. doi:10.1136/ bmjopen-2020-042627

- Prepublication history for this paper is available online. To view these files, please visit the journal online (http://dx.doi. org/10.1136/bmjopen-2020042627).

Received 09 July 2020 Revised 28 December 2020 Accepted 09 February 2021

D Check for updates

(C) Author(s) (or their employer(s)) 2021. Re-use permitted under CC BY-NC. No commercial re-use. See rights and permissions. Published by BMJ.

Department of Podiatry, University of Seville, Seville, Spain

Correspondence to Dr María Reina-Bueno; mreina1@us.es

\section{ABSTRACT}

Introduction Systemic lupus erythaematosus (SLE) is a chronic autoimmune disease of heterogeneous involvement. The disease may affect feet with a high prevalence of symptoms such as, for example, pain, forefoot and rearfoot deformities, and biomechanics dysfunctions. Custom-made foot orthoses (CMF0) have been previously reported to be effective in patients with other rheumatic diseases. However, as far as the authors know, there exist no studies about their effectiveness in SLE. This study aims at determining the effect of CMFO versus placebo flat cushioning insoles on pain, foot functionality, fatigue and quality of life in patients with SLE. Methods and analysis A randomised controlled trial would compare the effects of (1) CMFO and group B, which received a placebo, flat cushioning insoles, for 3 months. The main outcome measures are foot pain, foot functionality and foot-related disability. The secondary outcome measures are fatigue and quality of life.

Ethics and dissemination The study has been approved by the Portal de Ética de la Investigación Biomédica de Andalucía ethical committee 1494-N-19. The results will be disseminated regardless of the magnitude or direction of effect.

Trial registartion number Clinicaltrials.gov identifier NCT04098055.

\section{INTRODUCTION}

Systemic lupus erythaematosus (SLE) is a chronic autoimmune disease that causes heterogeneous clinical manifestations. The world incidence is estimated between 1:100000 and 10:100 000, ${ }^{1}$ and 9:10000 in Spain. ${ }^{2}$ It can affect any organ, including skin, joints, kidney and others. ${ }^{1}$ Musculoskeletal problems are estimated in $95 \%$ of cases during the course of the disease, with arthropathy being one of the earliest clinical manifestations. Joints may be affected in $90 \%$ of patients, and in some cases there exists symmetrical polyarthritis of small joints with deformities similar to rheumatoid arthritis, but non erosive. ${ }^{3}$
Strengths and limitations of this study

- First study that measures the effect on pain and function of custom-made foot orthoses (CMFO) in patients with systemic lupus erythaematosus (SLE).

- First study that measures the effect on quality of life of CMFO in patients with SLE.

- The follow-up by telephone emphasises the instructions given to participants.

- Blinded design limits internal and external bias.

- The main limitation is that patients wear different type of shoes.

It has been reported that $67 \%$ of patients with SLE have foot manifestations, as foot pain, deformities and biomechanical alterations. ${ }^{4}$ Also collagen alterations that may cause tendon rupture have been observed, and deformities related to Jaccoud's arthropathy that mainly affects the Achilles and patellar tendons. ${ }^{5} 6$ It is estimated that $10 \%-13 \%$ of patients also present tenosynovitis that may produce tibialis posterior tendonitis and plantar fasciitis. ${ }^{3}$

Cherry et $a l^{7}$ reported the frequency of lower limb or foot and ankle complications in 182 participants with SLE from the UK. A quantitative, cross-sectional, self-reported survey design was used. Eighty-seven per cent of the participants reported having experienced some form of lower limb musculoskeletal complication. The most frequently reported complication was joint pain $(80 \%$; $\mathrm{n}=145)$. A previous or current episode of loss of function, such that it was difficult or impossible to walk, was reported by $37 \%$ and $12 \%$, respectively.

Otter et $a l^{8}$ found that $77 \%$ of the participants responding to a questionnaire reported foot pain during the disease, which may have a negative impact on their quality of life and 
well-being. All regions of the foot were involved, the hindfoot and ankles being the most troublesome $(32 \%$ and $30 \%$, respectively). In another study that included 20 patients with SLE, it was observed that forefoot manifestations were highly prevalent, with the first metatarsophalangeal joint being affected in $80 \%$ of the cases. Moreover, moderate foot-related impairment was reported by $95 \%$ of the participants. ${ }^{9}$

Other studies have shown foot function changes in other systemic conditions using a custom device, compared with placebos. ${ }^{10-12}$ There are multiple references describing the convenience of using foot orthoses for the treatment of foot deformities caused by rheumatoid arthritis. ${ }^{13-30}$ SLE is an rheumatological disease showing similar foot affection that rheumatoid arthritis. In Otter $e t a l \mathrm{~s}^{8}$ study, $22 \%$ of the participants had been prescribed insoles, but half of them had stopped using this treatment. In spite of the prevalence of foot symptoms in people with SLE being high, the authors have not found clinical studies that address the effectiveness of orthopaedic treatment for foot problems. Therefore, this study aims at determining the effect of custom-made foot orthoses versus placebo flat cushioning insoles on pain, foot functionality, fatigue and quality of life in patients with LES. The authors hypothesised that patients with SLE will show significantly greater improvement in these variables when treated with custom-made foot orthoses, compared with those who wore a placebo.

\section{METHODS AND ANALYSIS}

Study design and setting

A single-centre randomised controlled clinical trial has been designed according to the Consolidated Standards of Reporting Trials (CONSORT) guidelines. ${ }^{31} \mathrm{~A}$ CONSORT flow diagram is presented (figure 1).

The design was carried out by the research group but was previously presented to the management of autoimmune and lupic association of Seville (ALUSEVILLA), in order to agree on aspects to improve it. The patients will be recruited from the following patient associations: ALUSEVILLA, Autoimmune and lupus association of Málaga, Lupus association of Cantabria, Lupus association of Galicia and Spanish Lupus Federation that includes

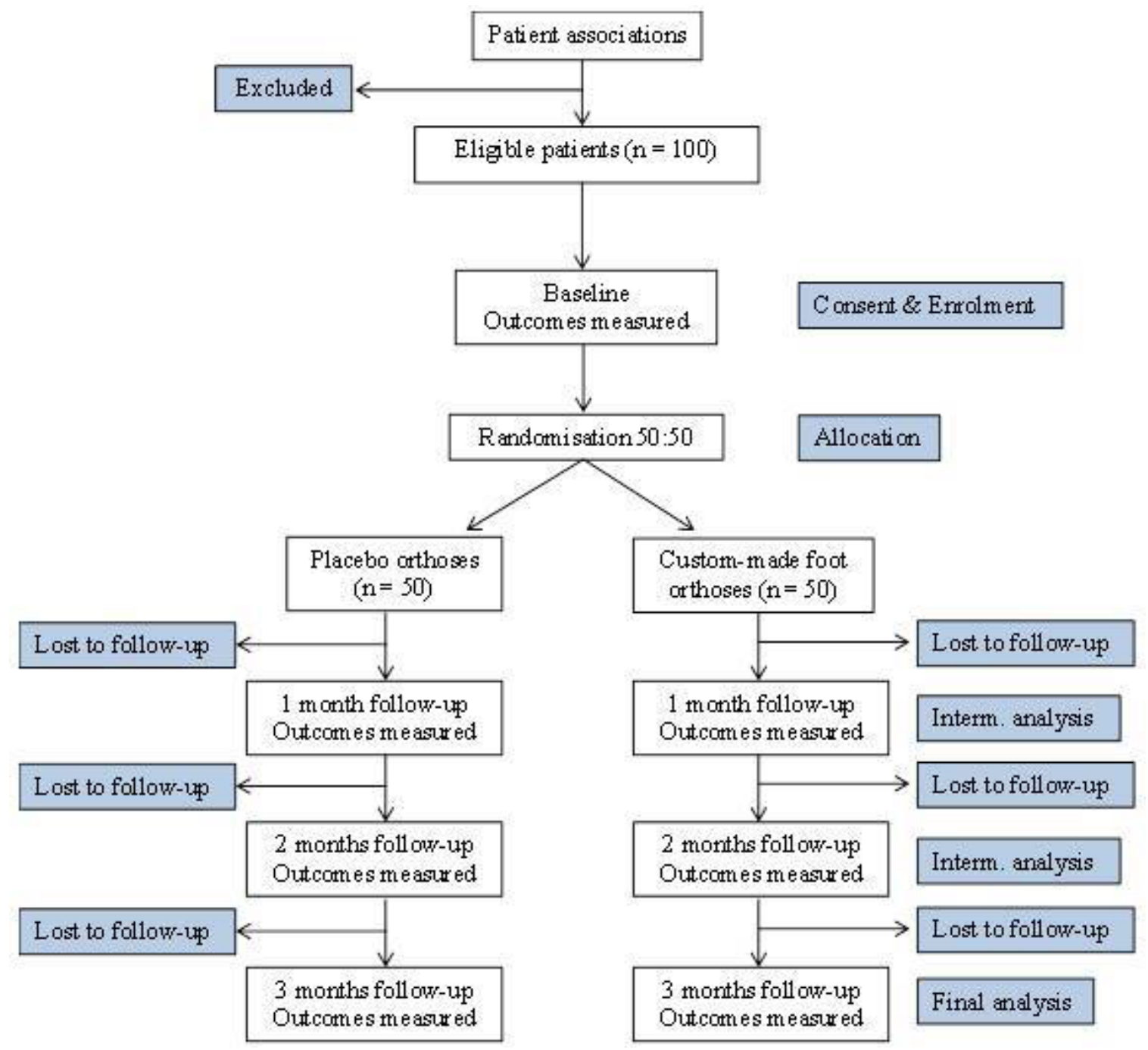

Figure 1 Consolidated Standards of Reporting Trials flow diagram outlining the flow of participants through the trial. 
all national associations. The data will be gathered in the Clinical Area of Podiatry of the University of Seville, and private clinics in Córdoba and Jerez de la Frontera (Beiman Córdoba and Beiman Jerez). The research team will get in contact with the persons in charge of those associations and they will inform the participants via their websites.

\section{Eligibility criteria}

The inclusion criteria will be: being between 18 and 70 years old, having a medical diagnosis of SLE according to the 2019 European League Against Rheumatism/American College of Rheumatology classification, ${ }^{32}$ foot involvement with a self-reported pain with a minimum threshold score of $\geq 3$, measured using the Numeric Pain Rating Scale (NPRS) and no foot orthotic treatment for 30 days prior to the study. The patients will be excluded if they refuse to use appropriated and healthy footwear for foot-made orthosis (with no more than a $3 \mathrm{~cm}$ drop, wide and spacious with removable, laced or velcro insole, good posterior buttress, medial-lateral stability and flexibility in metatarsophalangeal dorsoflexion). These criteria will be assessed by an examiner.

Patient is experiencing a flare or with foot pain $<3$ or will be excluded. Patients having ulcers, foot skin involvement (wound or injury), diabetes mellitus, pregnancy, neurological problems, cognitive deterioration, previous osteoarticular foot surgery, concomitant inflammatory arthritis or a need for walking assistance will be excluded from the study. Also, participants will be excluded if they refused to change their footwear to use the foot orthoses.

\section{Interventions}

All participants who voluntarily take part in the study will be given an informative document where the nature, objectives and potential risks of the investigation are explained. When they have read it and any doubts have been resolved, the informed consent will be given for them to sign.

First, clinical and demographic data will be collected, including age, gender, weight, height, years since diagnosis and current pharmacological management. Patients with SLE will be explored and the Foot Posture Index (FPI) (a validated method for quantifying standing foot posture), and the Manchester scale for hallux valgus will be recorded for both the right and left foot.

Standard podiatric care will be provided to the patients at the assessment session and when they finished the follow-up 3 months later, if needed. Participants will be requested not to have further foot interventions.

After the biomechanical examination, phenolic foam moulds will be made of the patient's feet under weightbearing conditions. The patient's foot will be manipulated before being introduced into the phenolic foam to place the subtalar joint in the most neutral position possible, always maintaining the forefoot plantar plane parallel to the floor.
In the case of FPI values between +6 and +12 , the examiner will hold the distal third of the participant's leg and apply external rotational force to the leg until the mirror of the podoscope shows incipient loss of the first ray footprint, that is, that the first metatarsal begins to elevate. At that moment, the examiner will stop applying external rotational force to the leg. This manoeuvre will be repeated several times by the examiner, as the external rotation applied to the foot in contact with the glass will be the same as that applied when introducing the foot into the phenolic foam. The same procedure but with internal rotation of the leg will be carried out for FPI values between -12 and -1 . For feet with FPI values between 0 and +5 , the examiner's hands will apply resistance against pronation or supination to maintain the foot in a normal posture.

In a standing position, the podiatrist will verify the fitting of the orthosis to the foot, and for group A, checking medial/lateral control and posterior stability. Then the insoles will be set into shoes. Finally, the participants will be asked is the insoles cause them pain and discomfort in every session. Both interventions have the same protocol.

The software AleatorMetod.xls, available at www4. ujaen.es/ mramos/EPIP/AleatorMetod.xls, will be used to randomly allocate the participants into two groups, and this allocation will be concealed in envelopes. The simple randomisation process will be carried out according to the order of appointment, so that the first patient treated will be number 1 . The participants themselves will choose the day and time of the meeting within an established time without knowing the order number that they have or the corresponding random assignment. Group A will be the experimental group and will receive the custom-made foot orthoses that consist of a polypropylene layer of $3 \mathrm{~mm}$ from heel to just proximal to the metatarsal heads, and an upper sheet of $5 \mathrm{~mm}, 30$ Shore A polyethylene foam. These orthoses will be obtained from the moulds of the participants' feet. Group B will be the control group and will be given the placebo treatment; that is, a flat insole made of the same material as the upper layer of the foot orthoses used for group A, with the sole aim of providing cushioning but not functional control. Both groups will be followed up for 3 months, and the data related to foot pain, foot function and quality of life will be recorded monthly.

\section{Primary outcomes measures}

The primary endpoint is pain and foot functionality, measured by the NPRS and Foot Function Index (FFI).

Self-reported pain intensity over the last month will be assessed with an 11-point NPRS with $0=$ no pain to $10=$ pain as bad as it can be..$^{33}$ Pain days will also be recorded as the number of days on which the patient felt foot pain in the previous week by assigning a whole number between 0 and 7.

Foot functionality will be measured using the FFI. ${ }^{34}$ This is a questionnaire with 23 items that are divided into three domains: foot pain, disability and functional 


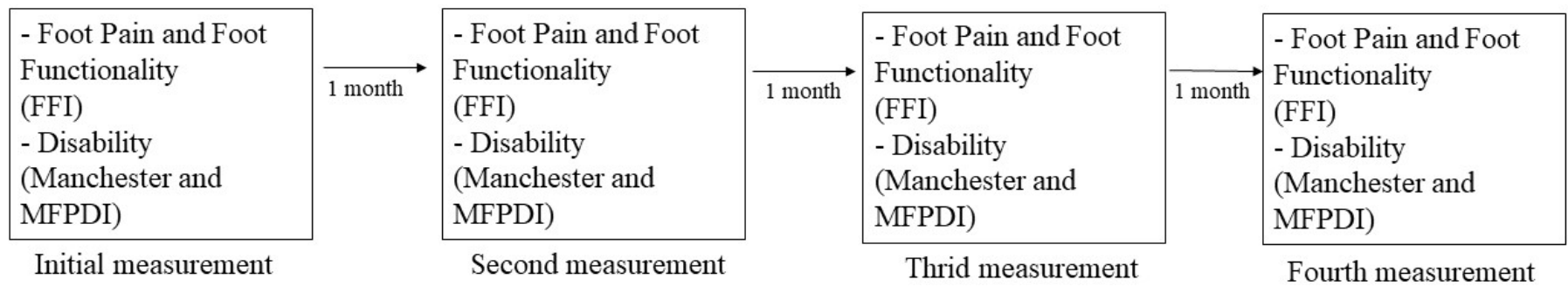

Figure 2 Clinical measurements emplacement: primary outcomes measures. FFI, Foot Function Index; MFPDI, Manchester Foot Pain and Disability Index.

limitation. The values range from 0 and 100, with higher values corresponding to greater pain, disability and limitation.

Disability related to foot pain will be measured using the Manchester Foot Pain and Disability Index. ${ }^{35}$ The values of this index range from 0 to 38, with higher values corresponding to greater disability (figure 2).

\section{Secondary outcomes measures}

The secondary outcome measures are fatigue and quality of life.

To evaluate the intensity of fatigue, the participants will be asked to complete the Fatigue Severity Scale (FSS). ${ }^{36}$ The FSS consists of nine items with a 7-point Likert-type response format indicating the degree of agreement with each statement. The scoring is done by calculating the average response to the questions (adding up all the answers and dividing by nine). Higher scores mean greater fatigue severity.

The SF-12 questionnaire will be used to collect data about the quality of life. ${ }^{37}$ This has values between 0 and 100 , with higher values corresponding to a lower quality of life.

The Spanish versions of these tools have demonstrated adequate values related to their reliability and validity ${ }^{33-37}$ (figure 3).

\section{Blinding and monitoring}

All the participants will be blinded, as they will not know what group they belong to and the type of orthoses they will be given in the study (intervention or placebo). They will only be informed that the research will evaluate this treatment to manage pain in patients with SLE, similarly to others previously used in other studies. ${ }^{10}$ The participants will be requested to use the foot orthoses assigned 7 days a week for a minimum of 8 hours per day for 3 months. The researcher who performs the measurements at days 0, 30, 60 and 90 will not be the same researcher who conducts the randomisation, adapts the foot orthoses and gives them to the participants. Thus, they will be blind as well. The clinical variables will be measured in person at the beginning (baseline) and at the end of the follow-up period, and via phone calls at days 30 and 60 . Adverse events will be recorded as part of the monitoring and appropriate safety measures. The participants will be asked about any problems with the insoles and their adaptation to the footwear.

\section{Sample size}

The minimum sample size was calculated using the following formula to compare mean values between populations:

$$
\left.n=\left(2 s \wedge 2(\alpha / 2)+z \_\beta\right)\right) / d \wedge 2,
$$

where s2 is the sample variance, $\alpha$ is the type I error, $\beta$ is the type II error and $d$ is the minimum difference to be detected. According to a previous study where the effect of foot orthoses on foot pain was investigated on patients with rheumatoid arthritis, ${ }^{10}$ the variance of the Visual Analogue Scale for pain is equal to 400, and the

Secondary Outcomes Measures

\begin{tabular}{|c|c|c|c|c|c|c|}
\hline - Fatigue (FFS) & & - Fatigue (FFS) & & - Fatigue (FFS) & & - Fatigue (FFS) \\
\hline $\begin{array}{l}\text { - Quality of Life } \\
\text { (SF-12) }\end{array}$ & 1 month & $\begin{array}{l}\text { - Quality of Life } \\
\text { (SF-12) }\end{array}$ & 1 month & $\begin{array}{l}\text { - Quality of Life } \\
\text { (SF-12) }\end{array}$ & $\overrightarrow{1 \text { month }}$ & $\begin{array}{l}\text { - } \begin{array}{l}\text { Quality of Life } \\
\text { (SF-12) }\end{array}\end{array}$ \\
\hline
\end{tabular}

Figure 3 Clinical measurements emplacement: secondary outcomes measures. FFS, Fatigue Severity Scale. 
difference found is $16^{\prime \prime}$. Therefore, the following result was obtained:

$$
\begin{aligned}
& \left.n=\left(2 s \wedge 2\left(z_{(} / 2\right)+z_{-}\right)\right) / d \wedge 2=2400(1.96+0.84) \\
& \wedge 2 / 16 \wedge 2=24.525
\end{aligned}
$$

Thus, at least 25 people will be needed in each group to compare the mean values. In this study, 60 patients will be initially recruited, with 30 in each group, in consideration of possible losses similar to previous studies (about $22 \%)^{10}$

\section{Statistical analysis}

The analysis of the data will be carried out using the statistical software IBM SPSS Statistics 22 (IBM). The descriptive data will provide the mean values and the SD or the absolute frequencies and percentages depending on whether the variables are scalar or categorical. ShapiroWilk tests will be conducted for the inferential analysis to determine the most appropriate test to use. When the data show a normal distribution by groups, a t-test will be carried out for independent samples. Mann-Whitney's U test will be used for the independent samples when there is no normal distribution. Since the study variables will be measured four times, the tests will be conducted in pairs for related samples as well. The t-test will be used if the variables show a normal distribution in the four measurements, and Wilcoxon's signed-rank test will be used for related samples when they do not. When statistically significant differences are found according to the $p$ value, the effect size will be calculated using Cohen's d or Rosenthal's $r$ to analyse the magnitude of the differences. The differences will be classified according to the following for both parameters: below 0.2: no effect; 0.2-0.5: small effect; 0.5-0.8: medium effect; and above 0.8 : large effect. ${ }^{38}{ }^{39}$ The analyses will be based on an intention-to-treat data set.

In the case of the variables for which randomisation produces differences between groups at the initial assessment, repeated-measures analyses of covariance with the baseline scores as covariates will be performed to enable comparisons of postintervention data after adjusting for those differences in the initial scores.

The confidence level a priori is $95 \%$.

\section{Missing data}

For missing data (participant withdrawal or interim missing data), the convenience of several missing data models (ie, 'missing at random', 'missing completely at random' or 'missing not at random') will be classified by the trial personnel (according to available trial data). The most appropriate missing data model will be selected.

\section{Data monitoring}

Data monitoring will be evaluated in collaboration with an external statistician, who will also review all the issues related to data collection and analysis during the research process.

\section{Ethics and dissemination}

The study has obtained ethical approval from the committee of Portal de Ética de la Investigación Biomédica de Andalucía 1494-N-19. Clinicaltrials.gov identifier NCT04098055.

Contributors MR-B, ICP-T, MdCV-B and PVM-M contributed to the conception of this study. PVM-M, GD-M and J-RO will do the statistical analysis. Data collectors will be collected by JMC-L, GD-M, MR-B, ICP-T and PVM-M. MR-B, PVM-M, JMC-L and ICP-T will be involved in the writing and in the review of the manuscript.

Funding The authors have not declared a specific grant for this research from any funding agency in the public, commercial or not-for-profit sectors.

Disclaimer The results will be disseminated regardless of the magnitude or direction of effect.

\section{Competing interests None declared.}

Patient and public involvement No patients are involved in setting the research question or the outcome measures, nor are they involved in the design or conduct of the study. No patients are asked to advise on the interpretation or writing up of results. There are no plans to disseminate the results of the research to the study's participants.

Patient consent for publication Not required.

Ethics approval Institutional review board that approved the protocol for the study: The study has been approved by the Portal de Ética de la Investigación Biomédica de Andalucía ethical committee (PEIBA) 1494-N-19. Clinicaltrials.gov identifier NCT03170947.

Provenance and peer review Not commissioned; externally peer reviewed.

Open access This is an open access article distributed in accordance with the Creative Commons Attribution Non Commercial (CC BY-NC 4.0) license, which permits others to distribute, remix, adapt, build upon this work non-commercially, and license their derivative works on different terms, provided the original work is properly cited, appropriate credit is given, any changes made indicated, and the use is non-commercial. See: http://creativecommons.org/licenses/by-nc/4.0/.

ORCID iD

María Reina-Bueno http://orcid.org/0000-0001-9316-8942

\section{REFERENCES}

1 Sociedad Española de Reumatología. Manual de Enfermedades reumáticas Ser, 2014. Available: https://www.ser.es/wp-content/ uploads/2015/10/Manual-SER.pdf

2 López-longo FJ, Calvo-Alén J. Registro nacional de pacientes Con lupus eritematoso sistémico de la Sociedad Española. Reum Clin 2014;10:17-24.

3 Pipili C, Sfritzeri A, Cholongitas E. Deforming arthropathy in systemic lupus erythematosus. Eur J Intern Med 2008;19:482-7.

4 Morales-Lozano R, Martínez-Barrio J, González-Fernández ML, et al The feet in systemic lupus erythematosus; are we underestimating their involvement and functional impact? Clin Exp Rheumatol 2016;34:609-17.

5 Alves EM, Macieira JC, Borba E, et al. Spontaneous tendon rupture in systemic lupus erythematosus: association with Jaccoud's arthropathy. Lupus 2010;19:247-54.

6 López Longo FJ. Artropatía de Jaccoud: Algo más que lupus. Semin la Fund Esp Reumatol 2011;12:36-41.

7 Cherry L, Alcacer-Pitarch B, Hopkinson N, et al. The prevalence of self-reported lower limb and foot health problems experienced by participants with systemic lupus erythematosus: results of a UK national survey. Lupus 2017;26:410-6.

8 Otter SJ, Kumar S, Gow P, et al. Patterns of foot complaints in systemic lupus erythematosus: a cross sectional survey. J Foot Ankle Res 2016;9:4-11.

9 Mukherjee S, Cherry L, Zarroug J, et al. A pilot investigation of the prevalence of US-detectable forefoot joint pathology and reported foot-related disability in participants with systemic lupus erythematosus. J Foot Ankle Res 2016;9:1-6.

10 Reina-Bueno M, Vázquez-Bautista MDC, Pérez-García S, et al. Effectiveness of custom-made foot orthoses in patients with rheumatoid arthritis: a randomized controlled trial. Clin Rehabil 2019;33:661-9. 
11 Rome K, Clark H, Gray J, et al. Clinical effectiveness and costeffectiveness of foot orthoses for people with established rheumatoid arthritis: an exploratory clinical trial. Scand J Rheumatol 2017:46:187-93

12 Castro-Méndez A, Munuera PV, Albornoz-Cabello M. The short-term effect of custom-made foot orthoses in subjects with excessive foot pronation and lower back pain: a randomized, double-blinded, clinical trial. Prosthet Orthot Int 2013;37:384-90.

13 Helliwell P, Woodburn J, Redmond A. The foot and ankle in rheumatoid arthritis. Edinburgh: Elsevier Ltd, 2007.

14 Davys HJ, Turner DE, Helliwell PS, et al. Debridement of plantar callosities in rheumatoid arthritis: a randomized controlled trial. Rheumatology 2005;44:207-10.

15 Gibson KS, Woodburn J, Porter D, et al. Functionally optimized orthoses for early rheumatoid arthritis foot disease: a study of mechanisms and patient experience. Arthritis Care Res 2014;66:1456-64.

16 Gijon-Nogueron G, Ramos-Petersen L, Ortega-Avila AB, et al. Effectiveness of foot orthoses in patients with rheumatoid arthritis related to disability and pain: a systematic review and meta-analysis. Qual Life Res 2018;27:3059-69.

17 Hennessy K, Burns J, Penkala S. Reducing plantar pressure in rheumatoid arthritis: a comparison of running versus off-the-shelf orthopaedic footwear. Clin Biomech 2007;22:917-23.

18 Hennessy K, Woodburn J, Steultjens MPM. Custom foot orthoses for rheumatoid arthritis: a systematic review. Arthritis Care Res 2012;64:311-20

19 Mejjad O, Vittecoq O, Pouplin S, et al. Foot orthotics decrease pain but do not improve gait in rheumatoid arthritis patients. Joint Bone Spine 2004;71:542-5.

20 Moreira E, Jones A, Oliveira HA, et al. Effectiveness of insole use in rheumatoid feet: a randomized controlled trial. Scand $J$ Rheumatol 2016;45:363-70.

21 Najafi B, Barnica E, Wrobel JS, et al. Dynamic plantar loading index: understanding the benefit of custom foot orthoses for painful pes cavus. J Biomech 2012;45:1705-11.

22 Novak P, Burger H, Tomsic M, et al. Influence of foot orthoses on plantar pressures, foot pain and walking ability of rheumatoid arthritis patients--a randomised controlled study. Disabil Rehabil 2009;31:638-45.

23 Budiman-Mak E, Conrad KJ, Roach KE, et al. Can foot orthoses prevent hallux valgus deformity in rheumatoid arthritis? A randomized clinical trial. J Clin Rheumatol 1995;1:313-22.

24 Cameron-Fiddes V, Santos D. The use of 'off-the-shelf' foot orthoses in the reduction of foot symptoms in patients with early rheumatoid arthritis. Foot 2013;23:123-9.
25 Chalmers AC, Busby C, Goyert J, et al. Metatarsalgia and rheumatoid arthritis--a randomized, single blind, sequential trial comparing 2 types of foot orthoses and supportive shoes. $J$ Rheumatol 2000;27:1643-7.

26 Cho NS, Hwang JH, Chang HJ, et al. Randomized controlled trial for clinical effects of varying types of insoles combined with specialized shoes in patients with rheumatoid arthritis of the foot. Clin Rehabil 2009;23:512-21.

27 Conceição CSda, Gomes Neto M, Mendes SMD, et al. Systematic review and meta-analysis of effects of foot orthoses on pain and disability in rheumatoid arthritis patients. Disabil Rehabil 2015;37:1209-13.

28 Conrad KJ, Budiman-Mak E, Roach KE, et al. Impacts of foot orthoses on pain and disability in rheumatoid arthritics. J Clin Epidemiol 1996;49:1-7.

29 Cuesta Calleja R, Polo García A, González Fernández ML. Evidencia científica del tratamiento ortopodológico en pie afectado POR artritis reumatoide. Rev Int Cienc Podol 2018;12:15-26.

30 Egan M, Brosseau L, Farmer M, et al. Splints and orthosis for treating rheumatoid arthritis. Cochrane Database Syst Rev;34.

31 Argimón-Pallás JM, Jiménez-Villa J. Métodos de investigación clínica y epidemiológica. Elsevier: Barcelona:, 2013.

32 Fanouriakis A, Kostopoulou M, Alunno A. Update of the EULAR recommendations for the management of systemic lupus erythematosus. Ann Rheum Dis 2019;2019:736-45.

33 Farrar JT, Young JP, LaMoreaux L, et al. Clinical importance of changes in chronic pain intensity measured on an 11-point numerica pain rating scale. Pain 2001;94:149-58.

34 Paez-Moguer J, Budiman-Mak E, Cuesta-Vargas Al. Cross-cultural adaptation and validation of the foot function index to Spanish. Foot Ankle Surg 2014;20:34-9.

35 Gijon-Nogueron G, Ndosi M, Luque-Suarez A, et al. Cross-cultural adaptation and validation of the Manchester foot pain and disability index into Spanish. Qual Life Res 2014;23:571-9.

36 Krupp LB, LaRocca NG, Muir-Nash J, et al. The fatigue severity scale. Application to patients with multiple sclerosis and systemic lupus erythematosus. Arch Neurol 1989;46:1121-3 http://www.ncbi. nlm.nih.gov/pubmed/2803071

37 Monteagudo Piqueras O, Hernando Arizaleta L, Palomar Rodríguez JA. Normas poblacionales de referencia de la versión española del SF-12V2 para La Región de Murcia. Gaceta Sanitaria 2011:25:50-61.

38 Cohen J. Statistical power analysis for the behavioral sciences. Stat Power Anal Behav Sci 1988;2:567.

39 Rosenthal R, Rubin DB, simple A. A simple, general purpose display of magnitude of experimental effect. J Educ Psychol 1982;74:166-9. 